SPECTROSCOPY AND PHOTOMETRY OF FAINT GALAXIES:

HINTS AT THEIR EVOLUTION

\author{
Hyron Spinrad \\ Dept. of Astronomy \\ University of California, Berkeley
}

\title{
ABSTRACT
}

I discuss four optical methods to locate standard candles (giant E galaxies), which should eventually lead us to reasonably large samples at high z. Redshift criteria and determinations are briefly discussed. The observed faint galaxy colors fit well into a simple model of stellar (galactic) evolution for systems with star-formation confined to a short time interval. Finally a summary of the statistics of blue galaxies in Coma-like clusters (Butcher-Oemler effect) is presented and interpreted.

\section{A BEGINNING}

The motivating force which drives some observers to the long and tedious observation of faint galaxies, is the dual hope of obtaining some understanding of galaxy evolution and, of course, reaching for the "cosmological grail."

To begin a modern attack on the problem we need methods to select our standard candles. We have to get a reasonably large sample at high z. To push into the unknown world, at $z>0.7$ is not easy, and time spent deciding on selection criteria may be well-spent.

I can think of four ways to accomplish a selection of giant $\mathrm{E}$ galaxies--the canonical standard candle. Some of the selective criteria may be successful, and all are worth examination, as they lead to different biases. They are:

1) Use of the classic ensemble object--the brightest galaxy in a moderately-populous cluster, discovered optically. Of course, at $z>0.55$ we begin to have discovery problems, as the clusters begin to dip beyond the range of large Schmidt cameras with even IIIa-J/IIIa-F emulsions. One may have to locate them at random on deep reflector plates ( $<1 / 0$ sq. per plate); unfortunately extra-optical techniques to find these clusters have not yet paid off. Figure 1 illustrates a rich 


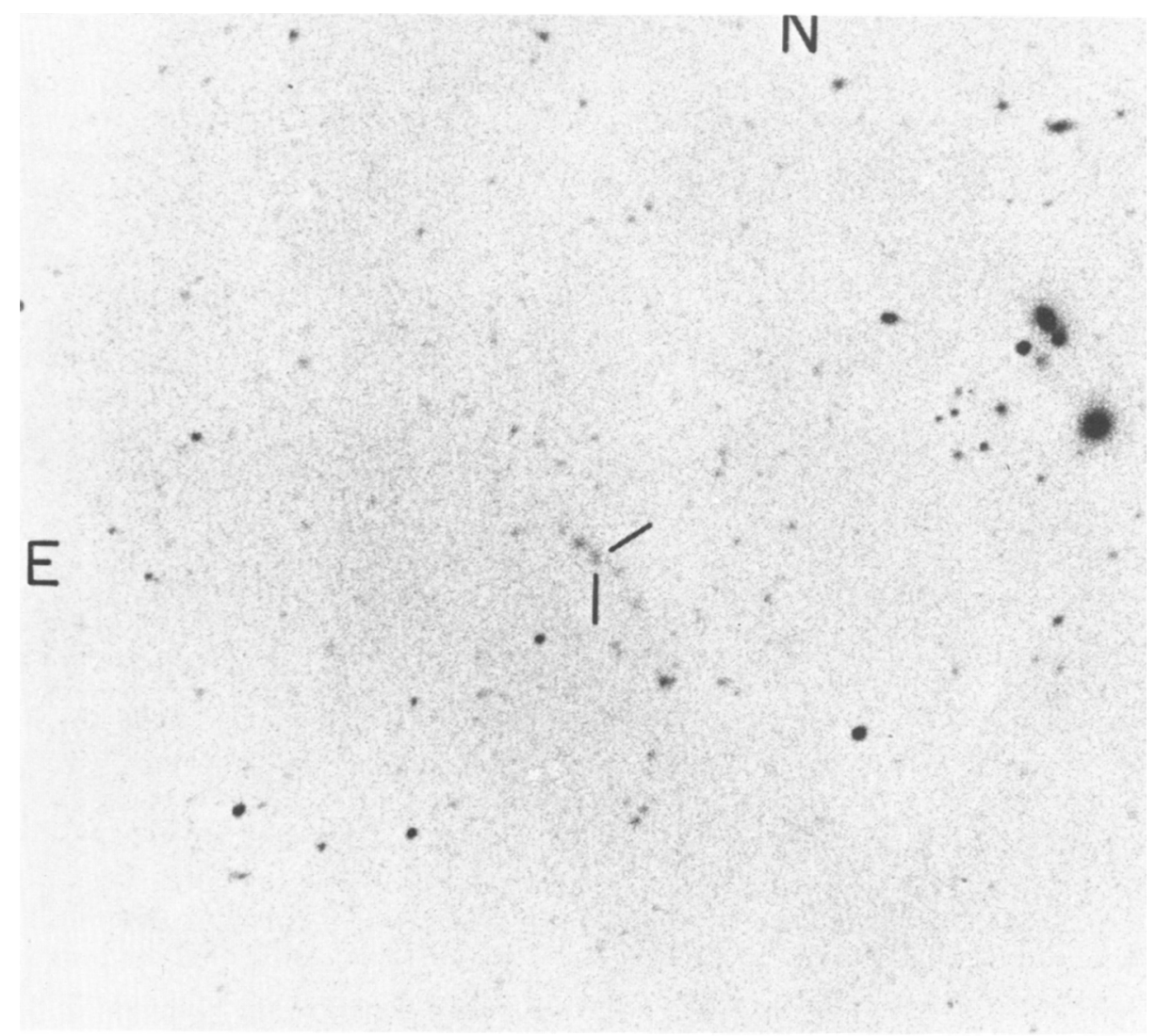

Figure 1. A print from a 4-m red photograph of the rich cluster $0015.9+1610$, at $z=0.541$. The 1st-ranked $\mathrm{E}$ galaxy is marked. 
galaxy cluster in SA 68 found by Richard Kron. The cluster redshift is $\mathbf{z}=0.541$ and the brightest galaxy has a red (F) magnitude of 20.4 . Locating random clusters at $\mathrm{z}>0.7$ will be difficult from the ground, even with a CCD array detector. From ST we should do better on red clusters by observing near $l_{\mu}$. The blue, very faint clusters are still a mystery!

2) We can also use radio galaxies as our probes to large distance. Hine and Longair (1979) show that over half the powerful 3CR classic double-lobed sources identified with galaxies have optical emission lines, which makes their redshift determination much easier. However, the non-thermal nuclear activity can make them optically untrustworthy in some cases, especially at emitted UV wavelengths (important observationally at large z) (c.f. Smith et al., 1979), so radio-galaxies are risky, albeit exciting candidates to include. Very few radio galaxies are underluminous (there is now one strange exception, 3C 258).

3) A new way to find standard candle ellipticals may be to look for very red galaxies, even in isolation. At $\mathrm{V}>19$ and color (J-F) $\geq 2 \mathrm{~m} 0$ all galaxies should be giant $\mathrm{E}$ systems, $0.4<z \leq 0.9$. This is because of a large K-correction in the J magnitude, the steeper ultraviolet continuum in the most luminous E's (Visvanathan and Sandage, 1977 ) and the modest color-evolution anticipated for them. These factors conspire to make luminous $\mathrm{E}$ galaxies in the mentioned red-shift range very red in (J-F) (Kron system). Of course, the high-z boundary is mode1-dependent.

A pilot program to obtain redshifts for several color-selected faint galaxies has begun at Lick Observatory. So far it seems promising, but again, somewhat demanding of spectroscopic observing time, as these red galaxies almost never show emission lines. Table 1 summarizes the start of this test; the magnitudes 1isted are all isophotal (to 26th in $\left.\mu_{\mathrm{r}}\right)$. The color-selected galaxies are almost, but not quite, up to the first-ranked cluster galaxy luminosity, which is about $\mathrm{M}_{\mathrm{v}}=-23.5$ (for $\left.\mathrm{H}_{\mathrm{O}}=50\right)$.

TABLE 1. Red-Color Galaxies - First Comparison

\begin{tabular}{llllc|l}
\hline Galaxy Name & Associates? & $\mathrm{F}_{\text {is }}^{26}$ & $\mathrm{~V}_{\text {is }}$ & $\mathrm{z}$ & $\Delta \mathrm{M}_{\mathrm{v}}^{*}(\mathrm{mag})$ \\
\hline SA 57, R3 & NO & 18.9 & 19.9 & 0.330 & +0.7 \\
Herc, 1718+50,R1 & A few & 19.8 & 20.8 & 0.450 & +0.6 \\
3C 295, g1 & Rich cluster & 18.4 & 19.4 & 0.461 & -0.8 \\
\hline
\end{tabular}

${ }^{*}$ Compared to ridge line of Kristian et al. (1978)

4) Finally, I suggest a new method using the reddest galaxies in clusters associated with quasars. Here we employ again the fact that E/SO galaxies around $0.4<z<0.9$ have a special color signature, and 


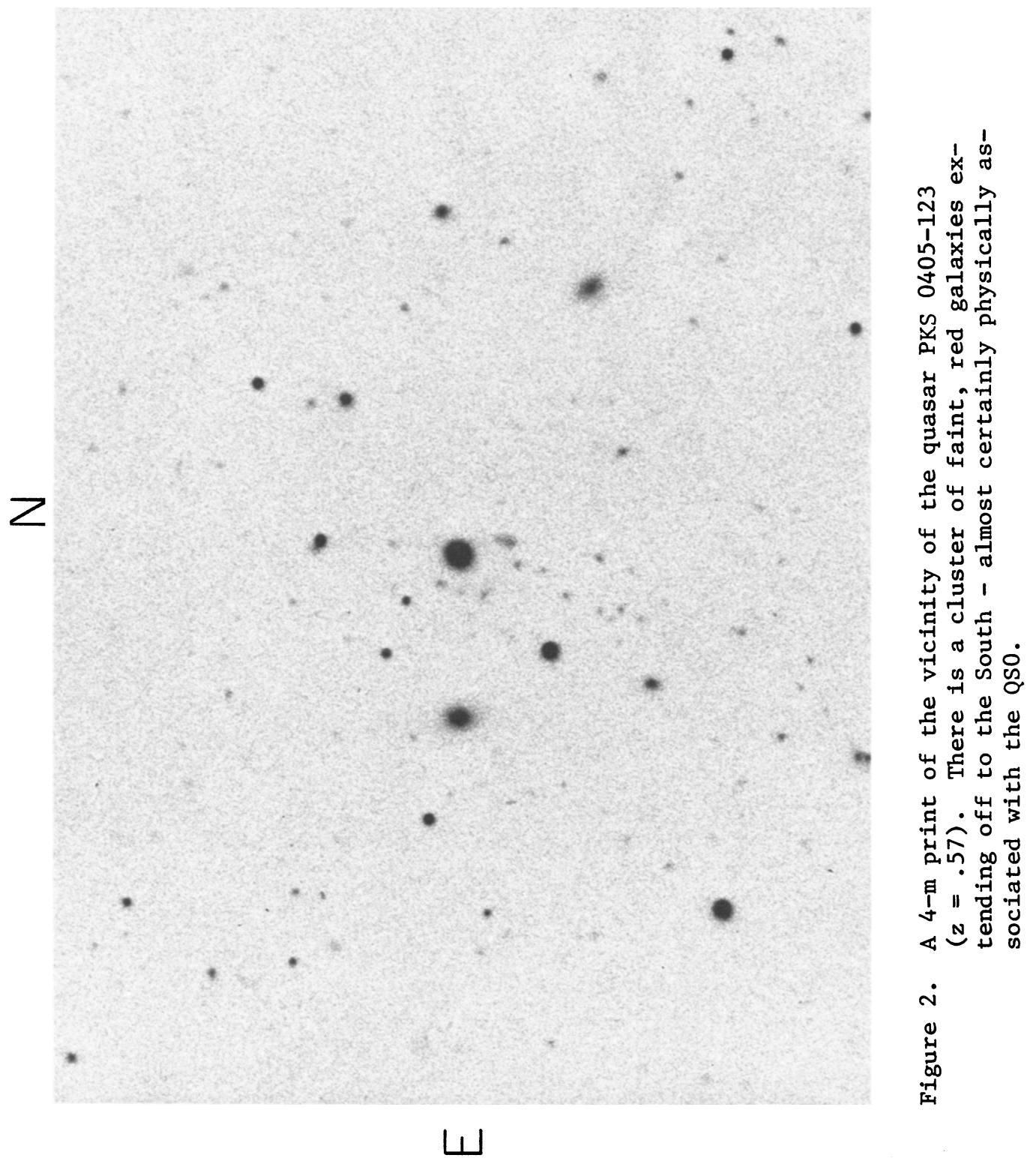


that very few random field objects are this red (Kron, 1978). Obvious1y a search for new quasar-clusters is in order, at $z_{e}>0.4$. We know from lower redshift quasars that normal galaxies are occasionally-tooften in concert (Gunn 1971, Stockton 1978, Wehinger and Wyckoff, 1978). Some more distant ones, for example PKS 0405-123 ( $z=0.574)$ also have apparent clusters and in this case (see Fig. 2) about half of the nearest faint galaxies have the colors demanded for E-galaxy membership. A test program of this selection procedure, with some controls, is planned for Cerro Tololo in January. This particular test brings forth a doctrinaire statement: we cannot let our former prejudices rob us of an opportunity to study the galaxies near quasars, from the ground or from ST.

\section{REDSHIFT CRITERIA AND DETERMINATIONS FOR LARGE-z GALAXIES}

Besides the long integrations required to determine galaxy redshifts even with large reflectors, simply settling upon redshift criteria presents new problems at $z>1.0$. The familiar $\lambda_{0} 4000$ break

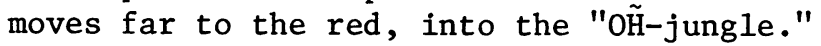

We now can, with the helpf of the IUE satellite ultra-violet spectra of stars and galaxies (c.f. Johnson 1979), spectrophotometry of two galaxies $(z \sim .5)$ to emitted $\lambda_{o} 2500$, and G. Bruzual's evolutionary predictions, make reasonable quantitative estimates about the UV spectra of normal large galaxies. In the case of a gas-poor E or So, seen in its relative youth ( $\sim 8$ billion years ago), we can anticipate the G-star discontinuities at $\lambda \lambda_{\circ} 2900,2640$, and 2420 (in order of $\lambda$ and importance). For galaxies with hotter stars (spirals) the situation is less clear, and $I$ am not sure if any Si-edge near $\lambda_{0} 1600$ will survive in the integral spectrum of an Sc galaxy. Probably the Lyman discontinuity at $\lambda_{0} 912$ will be a useful redshift criterion from ST (at $z \geq 0.3$ ), and conceivably even from the ground if primeval galaxies, $z \sim 5$, can be found.

A specific example of a redshift determined from the ultraviolet is the case I make for $3 \mathrm{C} \mathrm{427.1.} \mathrm{This} \mathrm{faint} \mathrm{radio} \mathrm{galaxy} \mathrm{has} \mathrm{no} \mathrm{emis-}$ sion lines, but does show the $\lambda \lambda_{0} 2900,2640$ edges, yielding $z=1.172$.

Emission-line galaxies at large-z could be more difficult than anticipated, too. The emitted ultraviolet doesn't have strong emission lines in HII region spectra; perhaps CIII] $\lambda_{0} 1909$ is the best feature. It will not be an easily applied criterion with the inevitably poor $\mathrm{S} / \mathrm{N}$ ratios obtained for faint galaxy spectra. The situation is very different from that which obtains in Seyfert nuclei and in QSO's which do have strong ultraviolet emission lines. In the latter case(s) the ionizing radiation is derived from a "hard" power-law spectrum extending far to the UV. 


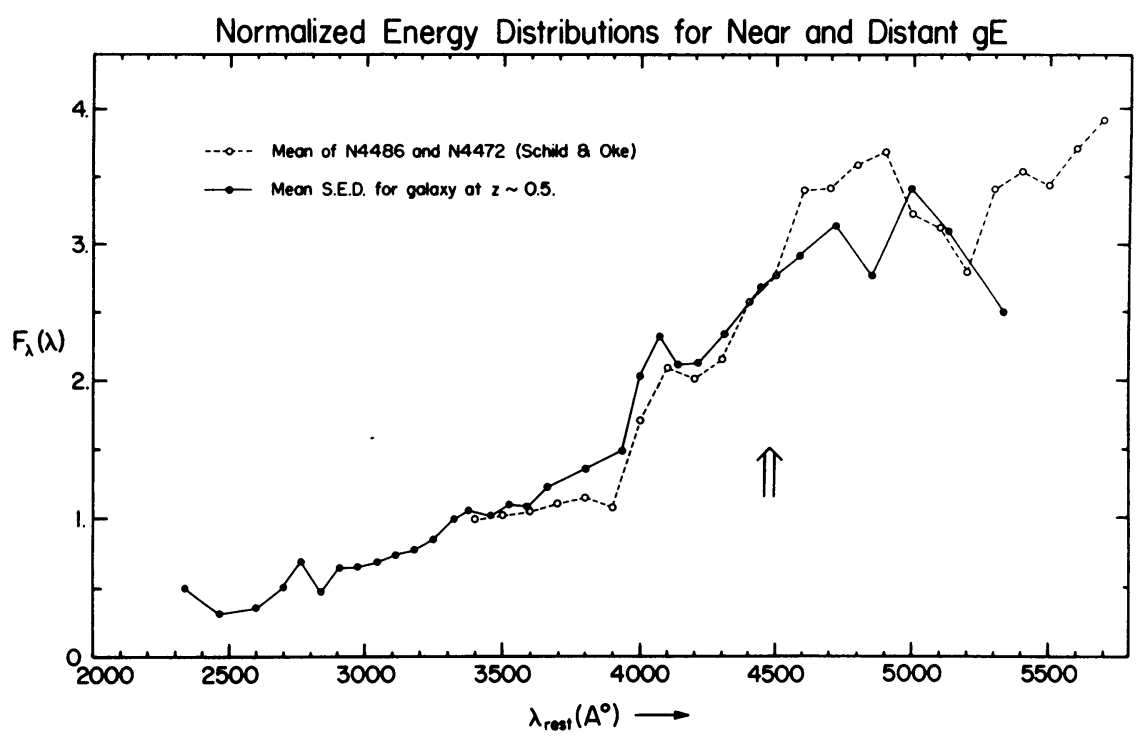

Figure 3. Normalized energy distribution for nearby (Virgo) and distant $(z=0.5)$ galaxies. The distant $E$ systems (an average of $3 \mathrm{C}$ 295, PKS 0400-64.3, and 3C 330, gal. 2) are somewhat bluer in their rest-frame spectra.

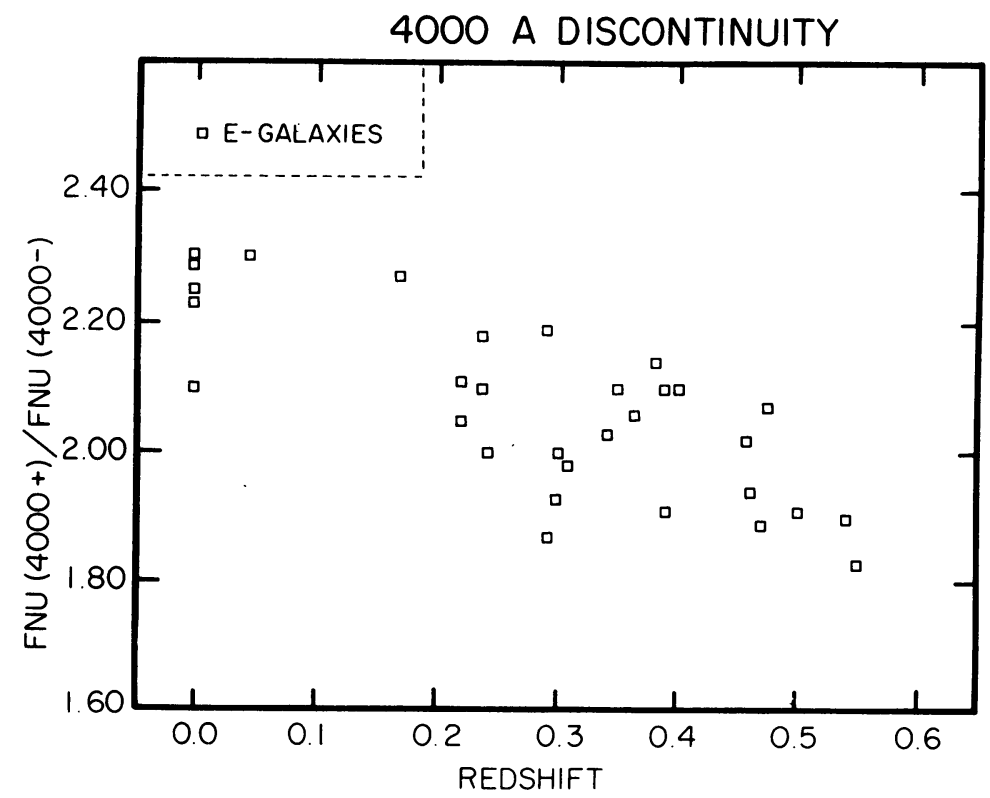

Figure 4. The decline of the amplitude of the $\lambda_{0} 4000$ break with redshift. Only "Thermal" E galaxy spectra have been used. 


\section{PREDICTIONS FOR EVOLVING MODEL GALAXIES}

As part of his ongoing UCB thesis, Gustavo Bruzual has given me permission to mention a very few results pertaining to galaxies at high$z$. The Bruzual models, patterned ater the assumptions made common by Tinsley, Larson, and others, make predictions of integral galaxy spectra and derive observables-magnitudes and colors, over the lookback times available to present and prospective future observation.

While these models have no claim to complete uniqueness in a domain where stellar abundance, age, mass-spectrum, and galaxy dynamics are all intertwined, a simple-minded interpretation can still be useful. With standard cosmological parameters of $\mathrm{H}_{\mathrm{O}}=50 \mathrm{~km}^{-1} \mathrm{Mpc}^{-1}$ and $\mathrm{q}_{\mathrm{o}} \cong 0.0$, galaxies are (arbitrarily) formed with a burst of star-formation 16 Gyrs ago. Another parameter describes the duration (exponentiation-time) of the star-forming-burst. A variety of observed $E$ galaxies, $0<z \leq 1.0$, fit the derived model colors over a wide wavelength interval, if the exponentiating decay time is roughly 1-2 Gyrs, a few galaxy collapse times. The last epoch of star-formation then occurred near $z=3$. The observed $(\mathrm{J}-\mathrm{F})_{\max }<2.3$ red color limit precludes a rapid single burst of stars at $z>4$ with no consequent "dribbles" of young stars, in any type galaxy considered.

\section{OBSERVED SPECTRAL EVOLUTION OF GIANT ELLIPTICALS}

An associated problem, detailed spectral evolution of elliptical galaxies, has a considerable literature. I reviewed it at Yale (Spinrad 1977); now the data is better, and we can claim that a positve change is detectable. Figure 3 shows the normalized $\left(\lambda_{0} 4500\right)$ energy distributions of two nearby giant $E^{\prime} s$ in Virgo, compared to the mean (rest-frame) s.e.d.'s for three galaxies at $\bar{z}=.5$. The distant (younger) galaxies are bluer at proper $\lambda_{0}<4300$, but only by about 0 m.15 in $(B-V)$. Soon IUE data will extend the comparison to $\lambda_{0} 2400$.

A more telling illustration of elliptical galaxy spectral evolution comes from data on the amplitude of the $\lambda_{0} 4000$ discontinuity. Figure 4 shows the break plotted vs. redshift; the steep decline in its amplitude is simply due to an increasing proportion of the light arising in turnoff stars of types F8-G2V, as we look at progressively "younger" E galaxies. The scatter is partly observational and apparently partly cosmic. Bruzual's models, with some effort, can model this evolutionary change rather well. Some differential model tests are possible if any high $\mathrm{S} / \mathrm{N}$ data on the $\lambda_{0} 4000$ break become available for cluster $\mathrm{E}^{\prime} \mathrm{s}$ at $z \sim 1.0$.

\section{DYNAMICAL EVOLUTION OF GALAXIES IN CLUSTERS}

Butcher and Oemler's (1978) discovery of substantial numbers of blue galaxies in concentrated, rich clusters has provided an exciting opportunity to observe the time-variation of the spiral content of Coma-like clusters--perhaps their residual dynamic collapse. Harvey 


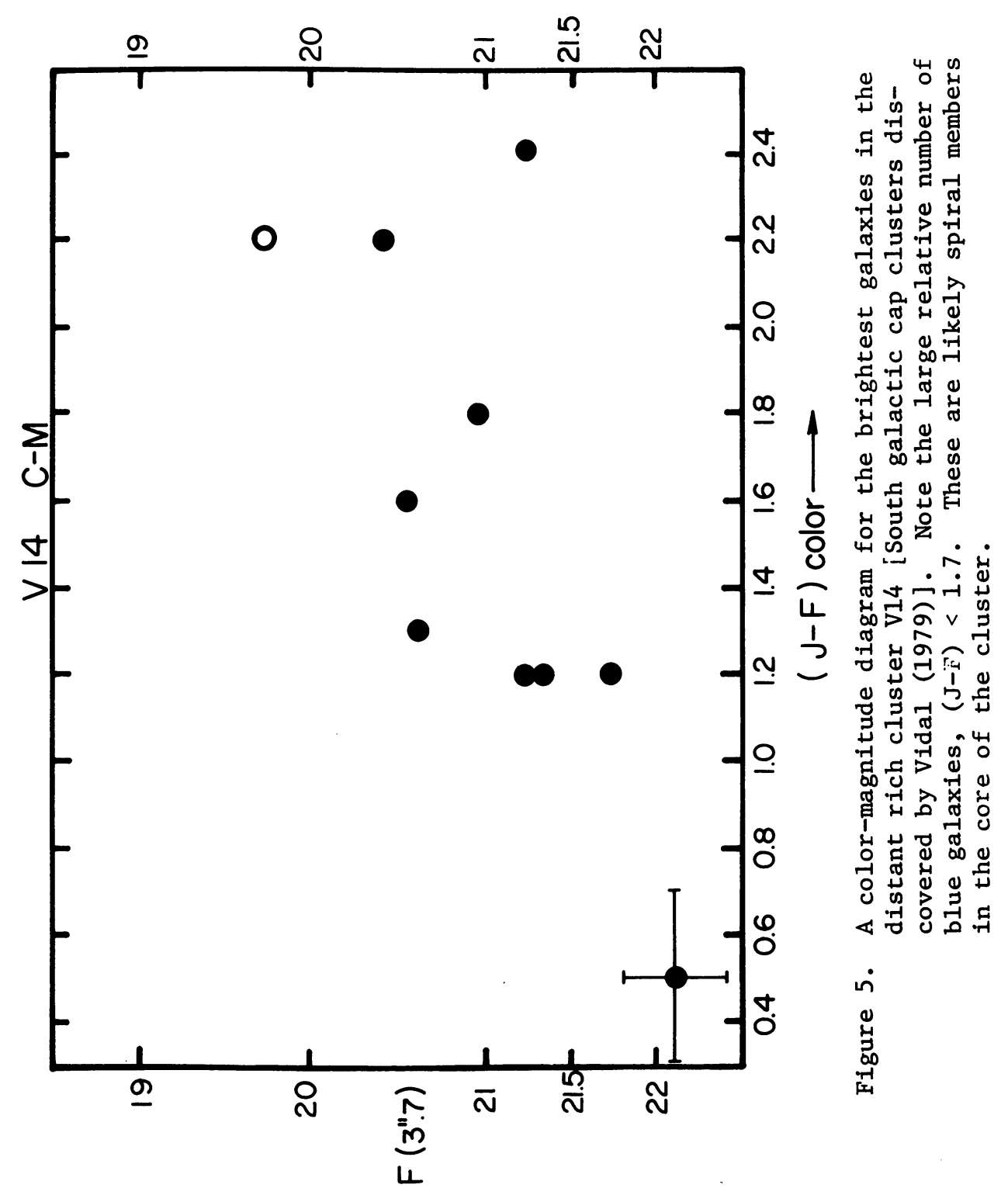


Butcher will discuss the KP work later in this volume, so my brief review will be encapsulated into a table which lists the fraction of (hopefully!) cluster members thought to be actively-star-forming (blue) spirals. There is a substantial trend with redshift.

Figure 5 shows a typical distant cluster C-M diagram from Berkeley PDS-photometry. V14 has a redshift of approximately 0.5 .

TABLE 2. Fraction of Luminous Cluster Galaxies at Least 0.4 Bluer Than Giant Ellipticals ( $>\mathrm{Sb}$ )

\begin{tabular}{llcl}
\hline Cluster & $\mathbf{z}$ & $\%$ Blue* & \multicolumn{1}{c}{ Notes } \\
\hline Coma & 0.02 & 5 & Standard concentrated cluster. \\
II 2w 1305.4 & 0.24 & 13 & Blue field galaxies removed. \\
Cor 0404 & 0.30 & 34 & Slightly less regular morphol. \\
$0949+44$ & 0.38 & 33 & Gunn-Oke cluster. \\
$0024+16$ & 0.39 & 27 & BO cluster \\
$3 \mathrm{C} \mathrm{295}$ & 0.46 & 33 & BO cluster, Berkeley photom. \\
V14 & $0.50 \pm$ & 55 & Redshift from sizes \& magns. \\
$1305+2952$ & $0.95:$ & 55 & Redshift not yet confirmed.
\end{tabular}

${ }^{*}$ In top $3^{\mathrm{m}}$ of $\mathrm{V}$ luminosity function.

A simple interpretation of this trend comes from a recent preprint by Larson, Tinsley, and Caldwell (1979), who suggest stripping of a spiral's gas-rich envelope occurs during a cluster's first collapse, and the subsequent decay of the SFR then produces present-day SO's. The process takes roughly 4 Gyrs, so a systematic trend in the spiral galaxy content in condensed clusters $(0.3 \leqslant z<0.6)$ is a natural consequence. We should then never see clusterrs with a larger fraction of blue galaxies then the local field percentage of late-type spirals $(\sim 60 \%)$.

Parenthetically, I should add, that at $z>1.5$, all galaxies may appear blue, no matter what the destiny of their morphologies. Could Kron's blue clusters fit into the scheme in such a manner? If so, they could be very useful future probes of cosmology and evolution. I would like to acknowledge many useful discussions with Gustavo Bruzual, David Koo, Rich Kron, Ivan King, and Bea Tinsley. I again thank the NSF for their support. 


\section{REFERENCES}

Butcher, H. and Oemler, A. 1978, Ap. J. 219, pp. 18-30.

Gunn, J. E. 1971, Ap. J. (Letters) 164, pp. L 113-118.

Hine, R. G., and Longair, M. S. 1979, M. N. 188, pp. 111-130.

Johnson, H. M. 1979, Ap. J. (Letters) 230, pp. L 131-136.

Kristian, J., Sandage, A. R. and Westpha1, J. A. 1978, Ap. J. 221, pp. 383-394.

Kron, R., Ph.D. Thesis, University of California, Berkeley.

Smith, H. E., Junkkarinen, V. T., Spinrad, H., Grueff, G., and Vigotti, M. 1979, Ap. J. 231, pp. 307-311.

Spinrad, H. 1977, Yale Conference on "The Evolution of Galaxies and Stellar Populations," pp. 301-338.

Stockton, A. 1978, Ap. J. 223, pp. 747-757.

Vidal, N. 1979, preprint.

Visvanathan, N., and Sandage, A. R. 1977, Ap. J. 216, pp. 214-226.

Wehinger, D., and Wyckoff, S. 1978, M. M. 184, pp. 335-340.

\section{DISCUSSION}

G. Burbidge: How sure are you of the redshifts of the three galaxies that you quoted in the beginning of your talk? In the case of the galaxies around the QSO with a redshift of 0.57 , will this influence you when you attempt to measure the galaxy redshift?

Spinrad: 1 . The 3 very red galaxies with $\mathrm{z}=0.33,0.48$ and 0.53 seem to have quite decent redshifts; the spectrum for the 2 brighter ones are respectable. The criteria for $z$ were conventional, the $\lambda_{0} 4000$ break and the inflexion at the G-band.

2. I would think the QSO at $z=0.57$ would influence the coice of grating tilt used for eventual spectroscopic tests on potential cluster galaxies. I also expect to report the results with honesty, no matter what the z-residuals are. 